\title{
PERFORMANCE COMPARISON OF ACTIVE AND SEMI-ACTIVE SMC AND LQR REGULATORS IN A QUARTER-CAR MODEL
}

\author{
Urszula Ferdek, Jan Łuczko \\ Cracow University of Technology, Faculty of Mechanical Engineering, Kraków, Poland \\ e-mail: uferdek@mech.pk.edu.pl; jluczko@mech.pk.edu.pl
}

\begin{abstract}
In this paper, an analysis is performed on a quarter-car model of car suspension with semi-active and active damper utilizing the sliding mode (SMC) and linear-quadratic control (LQR). The effect of control parameters and time delays in the transmission of control signal on the factors related to the safety and comfort of driving is investigated. The results obtained from numerical simulations are shown in form of frequency characteristics for several selected performance factors.
\end{abstract}

Keywords: active damping, semi-active damping, vibration reduction, car suspension

\section{Introduction}

For the purpose of checking the chosen concepts of active or semi-active control of car suspension, either quarter-car (Huang and Chen, 2006; Rajeswari and Lakshmi, 2008; Lin et al., 2009; Snamina et al., 2011) or, less often, half-car (Sam et al., 2008; Sapiński and Rosół, 2008) vehicle models are used. These are usually linear models with two- or four-degrees of freedom depending on the chosen type. They consist of both spring-supported masses (1/4 or $1 / 2$ car body) and non-spring supported ones (wheels with the reduced mass of the suspension system). The performance factor of the acting vibroisolation system should provide a compromise between the passengers' comfort level and their safety during driving (Łuczko and Ferdek, 2012). Several indexes of the driving comfort are introduced, either related to the displacements and velocities (Yoshimura et al., 2001; Chen and Huang, 2005; Sam and Osman, 2005) or to accelerations (Fischer and Isermann, 2004; Rao and Narayanan, 2009) of the so-called spring-supported mass, i.e. car body. Most often, the values used for this purpose are either mean or peak ones. The measure of the safety level is on the other hand derived form the net reaction or its dynamic component (Ahmadian and Vahdati, 2006), e.g. Eusam's index. With a decrease in the net reaction, the adhesion of the wheels and the steering decreases as well. The same happens to the performance of force transmission of driving and braking.

In the numerical simulations performed to analyze the behavior of a driving vehicle, the knowledge of the function describing the kinematic excitation acting on the vehicle is essential. Several different approaches to describing the irregularities in the road are presented in the literature. The road profile can be defined using a random function (Snamina et al., 2011) or a harmonic one of constant (Chen and Huang, 2005) or modulated frequency. If a vehicle is crossing some obstacles (e.g. bumps) either an impulse function of unit step type (Ahmadian and Vahdati, 2006) or other of more complex form (Lin et al., 2009; Luczko, 2011) are used.

In the active systems, several different types of control can be used. These are usually based on algorithms for the linear-quadratic regulation LQR (Rao and Narayanan, 2009; Orman and Snamina, 2009), PID regulation (Yildirim, 2004; Maciejewski, 2012) or sliding mode control (SMC) (Huang and Chen, 2006; Sam et al., 2008; Tomera, 2010). Theoretically, in the case of active damping, there are no limitations imposed on the function defining the control force. 
In the semi-active systems (Wu and Griffin, 1997; Fischer and Isermann, 2004; Liu et al., 2005) the basic restriction imposed on the control is based on the condition of preventing the introduction of energy to the system. This condition is fulfilled if the product of the control force and the velocity of spring-supported mass to the non-spring supported one (power) is less than zero. It is most often considered for the purpose of controlling damping properties of magneto-rheological dampers (Sapiński and Rosół, 2008; Makowski et al., 2011).

In this study, the emphasis is placed on analyzing the efficiency of two active control algorithms, utilizing LQR and SMC regulation, respectively. Additionally, an analysis of performance of semi-active systems, based on these regulators, is made. In the numerical simulations, the influence of time delay present between the control signal and the actuator is included.

\section{Quarter-car suspension model}

In this study, a quarter-car model is used as shown in Fig. 1. The motion of both masses: the non-spring-supported $m_{w}$ and the spring-supported one $m_{b}$ are defined by the variables $y_{w}$ and $y_{b}$. The displacement $w(t)$ is the given kinematic excitation, while $u(t)$ is the active or semi-active system influence on the mass elements $m_{w}$ and $m_{b}$. The parameters $k_{w}$ and $c_{w}$ define the spring and damping properties of the wheel, while the parameters $k_{b}$ and $c_{b}$ define the properties of the suspension system. Due to the negligible effect of damping of rubber on the results, this parameter is omitted in the analysis $\left(c_{w}=0\right)$.

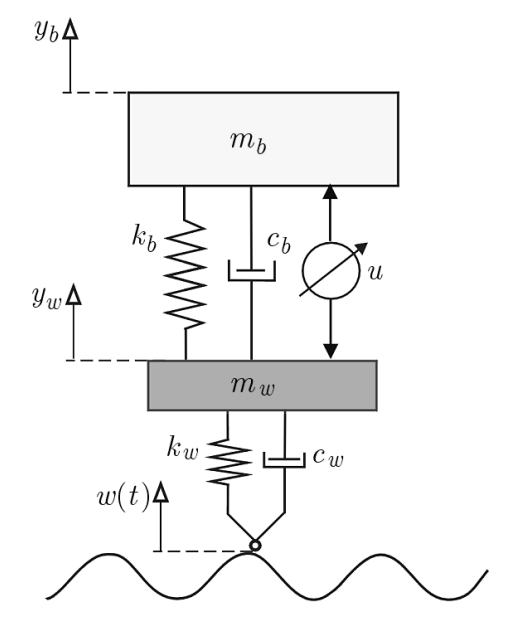

Fig. 1. Quarter-car model

Motion of the system around the static equilibrium position can be describen using the following matrix differential equation

$$
\mathbf{M} \ddot{\mathbf{y}}+\mathbf{C} \dot{\mathbf{y}}+\mathbf{K y}=\widetilde{\mathbf{B}} u+\widetilde{\mathbf{F}} w(t)
$$

where $\mathbf{y}=\left[y_{w}, y_{b}\right]^{\mathrm{T}}$. The matrices: mass $\mathbf{M}$, damping $\mathbf{C}$ and stiffness $\mathbf{K}$ are given as

$$
\mathbf{M}=\left[\begin{array}{cc}
m_{w} & 0 \\
0 & m_{b}
\end{array}\right] \quad \mathbf{C}=\left[\begin{array}{cc}
c_{b} & -c_{b} \\
-c_{b} & c_{b}
\end{array}\right] \quad \mathbf{K}=\left[\begin{array}{cc}
k_{w}+k_{b} & -k_{b} \\
-k_{b} & k_{b}
\end{array}\right]
$$

while the $\widetilde{\mathbf{B}}$ and $\widetilde{\mathbf{F}}$ vectors are of form

$$
\widetilde{\mathbf{B}}=\left[\begin{array}{c}
-1 \\
1
\end{array}\right] \quad \widetilde{\mathbf{F}}=\left[\begin{array}{c}
k_{w} \\
0
\end{array}\right]
$$


A modified state space vector is introduced, which includes generalized velocities

$$
\mathbf{x}=\left[\begin{array}{l}
\mathbf{x}_{1} \\
\mathbf{x}_{2}
\end{array}\right]=\left[\begin{array}{l}
\mathbf{y} \\
\dot{\mathbf{y}}
\end{array}\right]
$$

thus allowing one to write Eq. (2.1) in form of the first-order differential equation

$$
\dot{\mathbf{x}}=\mathbf{A} \mathbf{x}+\mathbf{B} u+\mathbf{F} w
$$

There is a relation between the matrix $\mathbf{A}$ and matrices present in Eq. (2.1)

$$
\mathbf{A}=\left[\begin{array}{cc}
\mathbf{0}^{(2 \times 2)} & \mathbf{I}^{(2 \times 2)} \\
-\mathbf{M}^{-1} \mathbf{K} & -\mathbf{M}^{-1} \mathbf{C}
\end{array}\right]
$$

in which the matrices $\mathbf{0}^{(2 \times 2)}$ and $\mathbf{I}^{(2 \times 2)}$ are respectively the empty and singular matrix of size $2 \times 2$. The same relation is for the matrices

$$
\mathbf{B}=\left[\begin{array}{c}
\mathbf{0}^{(2 \times 1)} \\
\mathbf{M}^{-1} \widetilde{\mathbf{B}}
\end{array}\right] \quad \mathbf{F}=\left[\begin{array}{c}
\mathbf{0}^{(2 \times 1)} \\
\mathbf{M}^{-1} \widetilde{\mathbf{F}}
\end{array}\right]
$$

where $\mathbf{0}^{(2 \times 1)}$ is an empty vector of length 2 .

\section{Linear-quadratic regulator (LQR)}

Linear-quadratic regulation is often used for vibration reduction (Rao and Narayanan, 2009; Orman and Snamina, 2009). The control signal generated by the controller depends on the actual state of the system

$$
u=-\mathbf{L x}
$$

The control if found using the minimal performance factor condition

$$
J=\int_{0}^{\infty}\left(\mathbf{x}^{\mathrm{T}} \mathbf{Q} \mathbf{x}+R u^{2}\right) d t
$$

in which $\mathbf{Q}$ is a positive half-defined weight matrix related to the state vector, while $R$ in the case of a single-input control is a positive weight coefficient When minimizing the car body vibration, it can be assumed that $\mathbf{Q}=\operatorname{diag}\left(0, q_{x}, 0, q_{v}\right)$. Such a form of the weight matrix should ensure the minimum of the second and fourth of the state vector variables, i.e. the displacement and velocity of the spring-supported mass. After the matrix $\mathbf{W}$, which is the solution to the Ricatti equation

$$
\mathbf{A}^{\mathrm{T}} \mathbf{W}+\mathbf{W A}-\mathbf{W B} R^{-1} \mathbf{B}^{\mathrm{T}} \mathbf{W}+\mathbf{Q}=\mathbf{0}
$$

is obtained, the matrix $\mathbf{L}$ (transposed vector) can be found from the formula

$$
\mathbf{L}=R^{-1} \mathbf{B}^{\mathrm{T}} \mathbf{W}
$$




\section{Sliding mode control (SMC)}

An alternative option for the active control of the car suspension is to use the algorithm of sliding mode control SMC (Yoshimura et al., 2001; Sam and Osman, 2005; Huang and Chen, 2006). During the process of regulation, two phases can be distinguished: the approach phase which lasts till the point that describes the dynamics of the system reaches the so-called "sliding plane", and the sliding phase. In the analyzed example, the sliding plane can be described using the formula

$$
S=\kappa_{x} e+\kappa_{v} \dot{e}
$$

in which $e$ is the regulation error. When minimizing the displacements of the spring-supported mass, this error can be assumed to be equal to the displacement of this mass, meaning $e=x_{2}=y_{b}$. Therefore, after the introduction of the transposed vector

$$
\mathbf{D}=\left[0, \kappa_{x}, 0, \kappa_{v}\right]
$$

the sliding plane equation can be written in the form

$$
S=\mathbf{D x}
$$

The approach to the sliding plane is ensured by the so-called compensation (equivalent) component of the control, which can be found from the relation

$$
\dot{S}=\mathbf{D} \dot{\mathbf{x}}=\mathbf{D}(\mathbf{A x}+\mathbf{B} u+\mathbf{F} w)=0
$$

The product $\mathbf{D F}$ of the transposed vector $\mathbf{D}$ and the vector $\mathbf{F}$ defined this way is equal to zero, which means that the equivalent component in this example does not depend on the excitation. If the model is well-known and no disturbance is present, the equivalent control defined by the formula

$$
u^{e q}(t)=-(\mathbf{D B})^{-1} \mathbf{D A x}
$$

compensating all the other forces acting on the spring-supported mass. In theory, the vibration of the system can be completely damped. Most often, the parameters of the model are not known, while the structure of the system is more complex, with the model being usually nonlinear. In a physical system, both the disturbances of the control signal and the time delay between the regulator and actuator are present. Due to these reasons, additional discontinuous control is added in form of a switching component: usw(t) (switching control)

$$
u^{s w}(t)=-K^{s w}(\mathbf{D B})^{-1} \operatorname{sgn}(S)=-K^{s w}(\mathbf{D B})^{-1} \operatorname{sgn}(\mathbf{D x})
$$

The final form of the control signal is given by the formula

$$
u(t)=-(\mathbf{D B})^{-1}\left[\mathbf{D A} \mathbf{x}+K^{s w} \operatorname{sgn}(\mathbf{D} \mathbf{x})\right.
$$

in which $K^{s w}$ is found based on the numerical simulation.

\section{Semi-active systems}

In semi-active systems, the realization of actual LQR or SMC control is not possible because of their active behavior. Therefore, these algorithms are usually modified to ensure that the momentary power is always negative (which means that no energy is introduced to the system). 
Additionally, see Orman and Snamina (2009), some sort of limitation is usually imposed on the control (clipped LQR). For such semi-active control systems, the control is found using the equation

$$
u^{\text {clipped }}=\varphi\left(u, u_{\max }, v\right)
$$

in which $\varphi$ is of form

$$
\varphi\left(u, u_{\max }, v\right)= \begin{cases}0 & v u \geqslant 0 \\ u & v u<0 \text { and }|u|<u_{\max } \\ u_{\max } & v u<0 \text { and } u \geqslant u_{\max } \\ -u_{\max } & v u<0 \text { and } u \leqslant-u_{\max }\end{cases}
$$

where $u$ is the control found from the condition of minimizing functional (3.2) or from Eq. (4.7), $u_{\max }$ is the maximal permissible value of control, while $v=v_{b}-v_{w}$ is the velocity of springsupported mass with relation to the non-spring-supported one. The effectiveness of semi-active damping can be estimated through numerical simulations.

\section{Results of numerical simulations}

The proposed algorithms of active and semi-active damping are based on the assumption that the model of the system is a precise description of the actual physical system. In practice, some sort of uncertainty of the model needs to be taken into account, mostly due to the simplified and usually linear description of the object and inaccurately determined parameters. Additionally, the disturbances and the time delay between the control signal and the actuator influence the performance of regulation. The analysis below is limited to finding the sensitivity of the designed regulator to the time delay only on the assumption that the acting force $U$ is related to the control signal $u$ by the equation of a first-order filter

$$
\dot{U}=-\sigma(U-u)
$$

where $\sigma$ is the inverse of the time constant. In the numerical calculations, the following values of parameters of the quarter-car model have been chosen: $m_{w}=28 \mathrm{~kg}, m_{b}=510 \mathrm{~kg}$, $k_{w}=180000 \mathrm{~N} / \mathrm{m}, k_{b}=20000 \mathrm{~N} / \mathrm{m}, c_{b}=1000 \mathrm{Ns} / \mathrm{m}$.

Below, the effect of parameters present in the control algorithms LQR and SMC on the indexes, describing the driving comfort and safety is presented. In order to measure the discomfort experienced by the passengers, both the displacement and velocity characteristics of the spring-supported mass are used. The driving safety is measured using the Eusam index $W_{E}$, which calculates the wheel-surface adhesion. Eusam's index is defined as a ratio of the maximum force to the static force acting on the wheel.

The values of indexes related to the driving comfort and safety reach maximum in different regions of excitation frequency. In the case of the vibration of frequency within the first vibration mode region, the car body displacements are dominant, which means that the driving comfort is worse. When the frequency of vibration is within the region close to the second natural mode, the displacements of car suspension are larger, and the wheel adhesion to the road is decreased. In order to evaluate the global performance of the regulator, it is advised to use either the frequency characteristics of the system or analyze the response of the system to the excitation of modulated frequency, e.g. the function "chirp". Below, the function of "chirp" type is used with a variable amplitude of the excitation. The proposed modification is introduced due to the fact that the frequency of excitation related to the velocity of the vehicle is increased, with an 
increase in the amplitude or, more precisely, with a decrease in the irregularities of the road. The noticeable effect of such a correction to the excitation are more realistic values of the Eusam index. Without this modification, when the amplitude of excitation is too high, the Eusam index is negative within the high frequency range (which denotes the loss of adhesion), and practically independent of the used vibroisolation system.

Further, it is assumed that with an increase in the excitation frequency, the amplitude decreases according to the formula

$$
a=\frac{\alpha_{1}}{\left(\alpha_{2}+\omega\right)^{2}}
$$

The value of coefficients $\alpha_{1}$ and $\alpha_{2}$ are found from the condition that the amplitude value close to the first resonance frequency $(\omega=8.396 \mathrm{rad} / \mathrm{s})$ is equal $\alpha_{0}=0.005 \mathrm{~m}$, and around the second resonance frequency $(\omega=84.39 \mathrm{rad} / \mathrm{s})$ the amplitude is $0.0004 \mathrm{~m}\left(\alpha_{1}=914.2631 \mathrm{~ms}^{2} / \mathrm{rad}^{2}\right.$ and $\left.\alpha_{2}=21.3806 \mathrm{rad} / \mathrm{s}\right)$. Additionally, it is assumed that the frequency increases proportionally to the square of time

$$
\omega=\omega_{1}+\varepsilon t^{2}=\omega_{1}+\left(\omega_{2}-\omega_{1}\right)\left(\frac{t}{T_{s i m}}\right)^{2}
$$

while in the region limited by the frequencies $\omega_{1}$ and $\omega_{2}\left(\omega_{1}=1 \mathrm{rad} / \mathrm{s}, \omega_{2}=100 \mathrm{rad} / \mathrm{s}\right)$ both resonance frequencies are present. The parameter $T_{\text {sim }}$ is the large enough duration of simulation. Figure 2 shows the kinematic excitation in the function of time

$$
w(t)=a \sin \left(\omega_{1} t+\varepsilon \frac{t^{3}}{3}\right)
$$

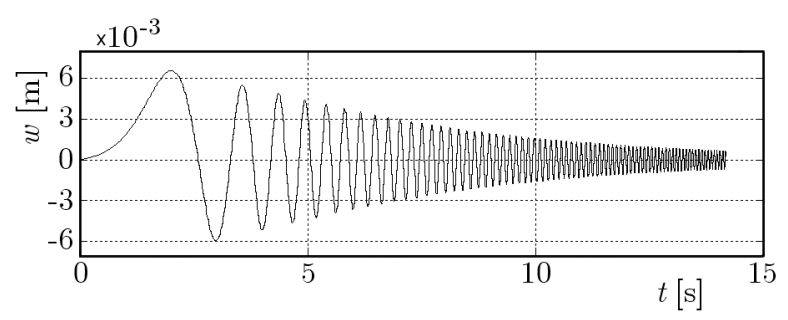

Fig. 2. Kinematic excitation $w(t)$

The effectiveness of both considered regulators depends on three parameters $\left(q_{x}, q_{v}\right.$ and $R$ for LQR, $\kappa_{x}, \kappa_{v}$ and $K^{s w}$ for SMC). As the units of the parameters in both these cases are different, it is better to use dimensionless control parameters defined as follows: $\alpha_{x}=q_{x} K_{x}^{2}$, $\alpha_{v}=q_{v} K_{v}^{2}, \rho=R K_{u}^{2}$ for LQR and $\beta_{x}=\kappa_{x} K_{x}, \beta_{v}=\kappa_{v} K_{v}, \gamma=K^{s w} / \omega_{0}$ for SMC, with: $K_{x}=a_{0}, K_{v}=\omega_{0} a_{0}, K_{u}=k_{b} a_{0}, \omega_{0}=\sqrt{k_{b} / m_{b}}$.

In the case of a linear-quadratic regulator, one of the parameters: $\alpha_{x}, \alpha_{v}$ or $\rho$ can be chosen arbitrarily due to the form of functional (3.2). In the numerical simulation presented here, the value $\alpha_{v}=1$ is chosen. The ratio between the parameters $\alpha_{x}$ and $\rho$ highly influences the results. With an increase in this ratio, the amplitudes of displacements, velocities and accelerations are decreased, especially within the range of the first resonance. Additionally, the Eusam index is decreased, which means that the wheel adhesion to the road is worse.

For the values $\alpha_{x}=100$ and $\rho=0.1$ in the wide frequency range, both the amplitude of vibration (Fig. 3a) and the Eusam index (Fig. 3b) are satisfactory. For example, within the range of the first resonance, a reduction in the displacement amplitude by eight times is visible $\left(x_{2 \max }<0.6 \mathrm{~mm}\right.$ for $\omega \approx 8.4 \mathrm{rad} / \mathrm{s}$ and $\left.a=5 \mathrm{~mm}\right)$, while in the range of the second resonance, the index $W_{E}>0.8$. 

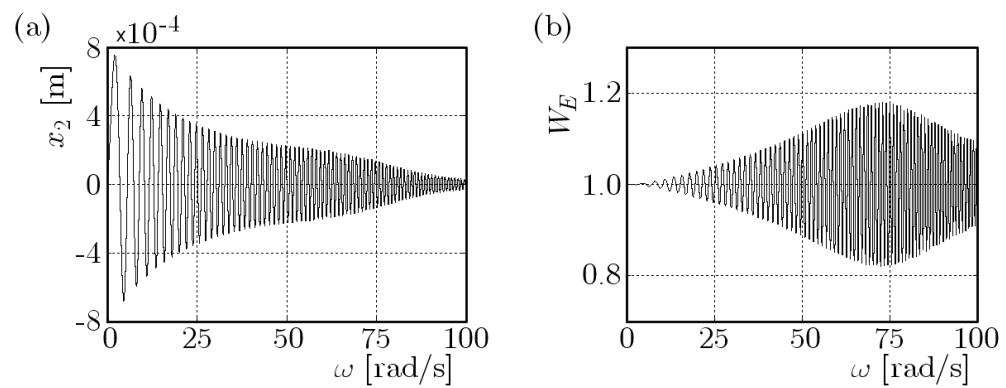

Fig. 3. LQR controller $\left(\alpha_{x}=100, \alpha_{v}=1, \rho=0.1, \sigma=100 \mathrm{~s}^{-1}\right)$ : (a) displacement $x_{2}$, (b) Eusam index $W_{E}$

In order to better illustrate the effect of control parameters on the selected frequency characteristics, in the following figures (Figs. 4, 5,6) the maximum (or minimal in the case of the Eusam index) values of the response of the system to the excitation defined by Eqs. (6.2)-(6.4) are shown.

Figure 4 shows the velocities (Fig. 4a) and the Eusam index (Fig. 4b) as functions of the excitation frequency acquired for the system with LQR for six different values of parameter $\alpha_{x}$ and $\alpha_{v}=1, \rho=0.1, \sigma=100 \mathrm{~s}^{-1}$. With an increase in the parameter $\alpha_{x}$, a decrease in the displacement, velocities and accelerations in the low-frequency range is noticeable, but the Eusam index (as well as the momentary power) is increased near the second resonance.
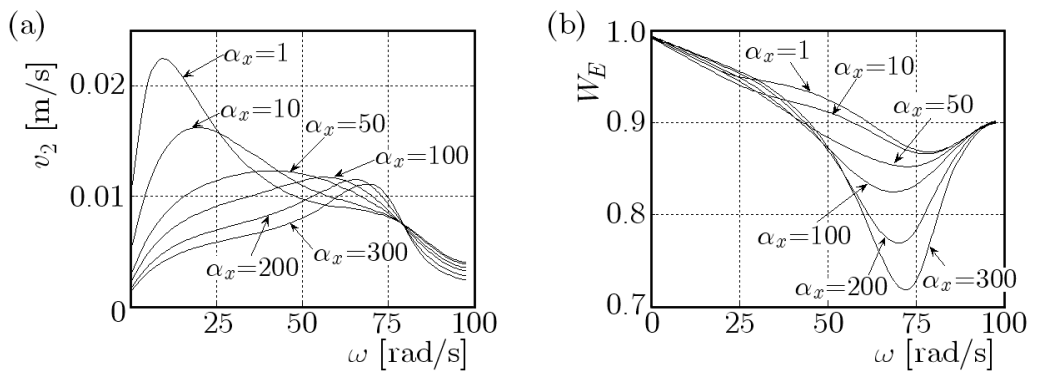

Fig. 4. LQR controller - effect of parameter $\alpha_{x}\left(\alpha_{v}=1, \rho=0.1\right.$ and $\left.\sigma=100 \mathrm{~s}^{-1}\right)$ : (a) velocity $v_{2}$,

(b) Eusam index $W_{E}$

Figure 5 shows the effect of the parameter $\rho$ on the frequency characteristics of the system. When the value $\rho$ decreases, both the velocities and displacements decrease as well (Fig. 5a), while the acceleration of the spring-supported mass are relatively low within the low-frequency range (Fig. 5b). As the lower values of the parameter $\rho$ require less restrictions imposed on the control signal, with a decreasing of its value, the control forces, Eusam index (Fig. 5c) and the momentary power (Fig. 5d) begin to rise (rapidly at $\rho<0.05$ ).

The effect of time delay between the control signal $u$ - force $U$, (Fig. 6; velocities and power), is notice within the high-frequency range. In a relatively wide range, with an increase in the parameter $\sigma$ (larger time delay), both the vibration of the spring-supported mass (Fig. 6a) and the value of dynamic reaction increase, which causes the minimum value of Eusam index to decrease. Within this range, high values of the power (Fig. 6b), denoting changes in energy introduced to the system, can be observed. Although the parameters of control are chosen to be optimal, the active system is not very effective in this range of frequencies.

Figure 7 shows the displacements and Eusam index for the SMC regulator obtained in the similar way as for LQR (Fig. 3). The numerical simulations are performed for $\beta_{x}=\beta_{v}=1$, $\gamma=0.1$ and $\sigma=100 \mathrm{~s}^{-1}$. The function $\operatorname{sgn}(S)$, present in Eq. (4.6), is approximated by a continuous function $2 / \pi \arctan (\eta S)$ with the parameter $\eta=100$. In the case of SMC regulator, much better vibration reduction can be accomplished (Fig. 7a) than for LQR (Fig. 3a) especially 

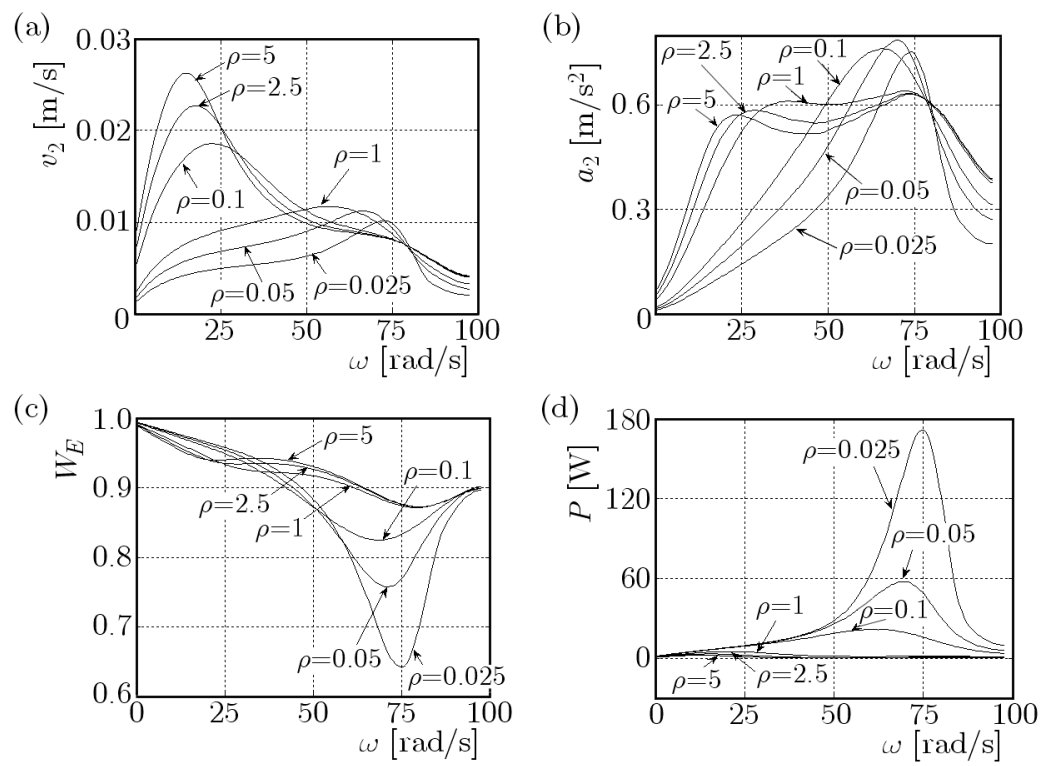

Fig. 5. LQR controller - effect of parameter $\rho\left(\alpha_{x}=100, \alpha_{v}=1, \sigma=100 \mathrm{~s}^{-1}\right)$ : (a) velocity $v_{2}$,

(b) acceleration $a_{2}$, (c) Eusam index $W_{E}$, (d) momentary power $P$
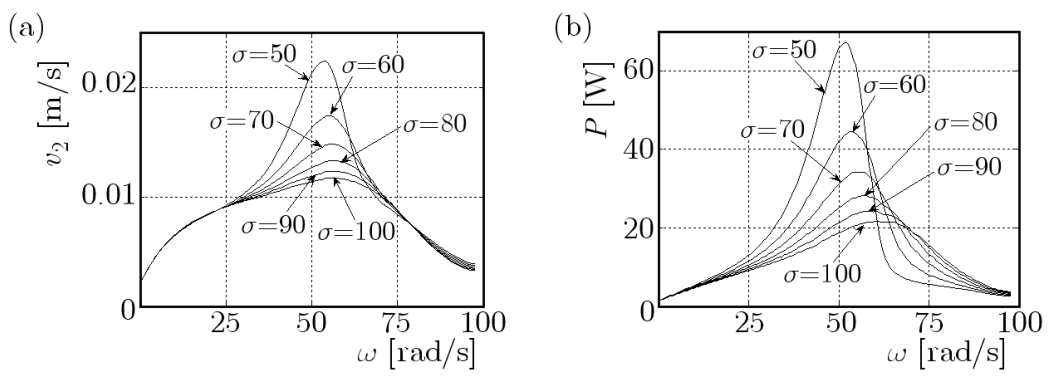

Fig. 6. LQR regulator - effect of parameter $\sigma\left(\alpha_{x}=100, \alpha_{v}=1, \rho=0.1\right)$ : (a) velocity $v_{2}$, (b) momentary power
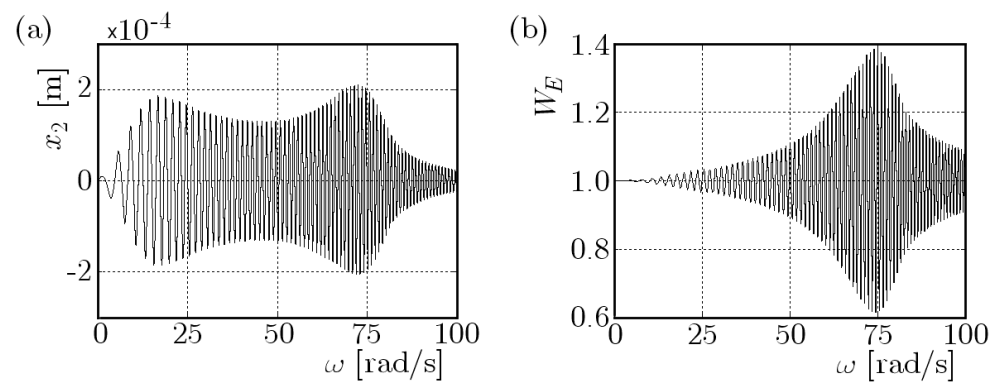

Fig. 7. SMC regulation $\left(\beta_{x}=\beta_{v}=1, \gamma=0.1, \sigma=100 \mathrm{~s}^{-1}\right)$ : (a) displacement $x_{2}$, (b) Eusam index $W_{E}$

in the low-frequency range. Similarly, as with LQR, within the high-frequency range both the Eusam index (Fig. 7b) and the momentary power (not shown here) are unsatisfactory.

Figure 8 shows the characteristics of the SMC regulator obtained for six different values of the parameter $\beta_{x}$. The effect of the parameter $\beta_{x}$ on the frequency characteristics is, apart from the slightly different plot, similar to the parameter $\alpha_{x}$ for LQR (Fig. 4). With an increase in $\beta_{x}$, the amplitude of vibration decreases in the lower frequency range. However, if this value is too high, the so-called effect of "chattering" might occur, which causes the characteristic of the system to greatly deteriorate in the high-frequency range. 
(a)

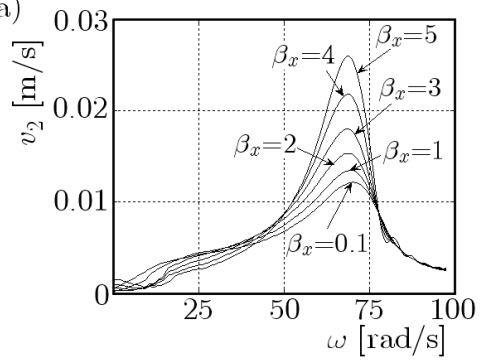

(b)

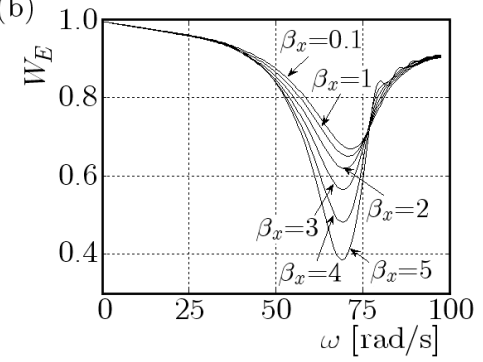

Fig. 8. SMC regulation - effect of parameter $\beta_{x}\left(\beta_{v}=1, \gamma=0.1, \sigma=100 \mathrm{~s}^{-1}\right)$ : (a) velocity $v_{2}$, (b) Eusam index $W_{E}$

(a)

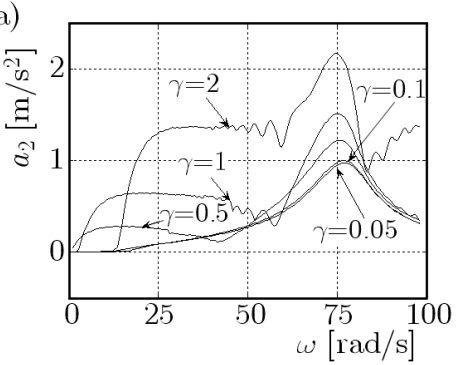

(b)

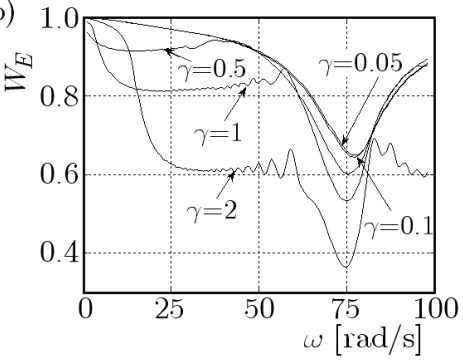

Fig. 9. SMC regulation - effect of parameter $\gamma\left(\beta_{x}=\beta_{v}=1, \sigma=100 \mathrm{~s}^{-1}\right)$ : (a) acceleration $a_{2}$, (b) Eusam index $W_{E}$

The "chattering" effect - the appearance of high frequency oscillation, is caused mostly by the switching component of control $u^{s w}(t)$, visible in the time plots. This effect is also the cause of irregular frequency characteristics plots. Figure 9a (maximum accelerations) and Fig. 9b (Eusam index) obtained for $\beta_{x}=\beta_{v}=1, \sigma=100 \mathrm{~s}^{-1}$ and five different values of $\gamma$ partially illustrate the effect of "chattering". From the analysis of numerous results of numerical simulations, a conclusion can made that with an increase in the parameter $\gamma$ (and also $\beta_{x}$ ) the vibration amplitudes decrease in the range of the first resonance, but for the excitation of high frequency, the maximal value of the control force is increased, while the minimal value of the Eusam index is decreased (Fig. 9b). Additionally, after exceeding certain values (for $\gamma>0.1$ ), a rapid rise in accelerations in noticeable (Fig. 9a) and the possibly of occuring of the "chattering" effect is high.

The frequency characteristics of the system depend also on the value of the parameter $\sigma$. This influence is visible in Fig. 10, obtained for six values of $\sigma$ and $\beta_{x}=\beta_{v}=1, \gamma=0.1$. With an increase in $\sigma$, the regulator is more effective in the low-frequency range (see Fig. 10a for velocities, Fig. 10b for accelerations), however its efficiency is greatly decreased in the second resonance range, and the power requirement is much higher, especially for high values of the parameter $\beta_{x}$ (for $\beta_{x}>1$ ).

Figure 11 shows the frequency characteristics of semi-active LQR (denoted as LQR-S) for $\alpha_{x}=\alpha_{v}=1$ and $\rho=0.1$, and also SMC (SMC-S) for $\beta_{x}=\beta_{v}=1$ and $\gamma=0.1$. Additionally, a characteristic of the system without regulator is presented (LIN).

For the parameters chosen as such, the efficiency of the semi-active damper is satisfactory both in terms of driving comfort (Fig. 11a) and safety (Fig. 11b). Within the range of the first resonance, both semi-active systems are thrice as effective as the passive system. In the high-frequency range on the other hand, the performance of semi-active and passive systems is compatible. Semi-active regulators, when compared to the active ones, are less vulnerable to changes in the parameter $\sigma$. In the calculations, these values have been assumed as $\sigma=100 \mathrm{~s}^{-1}$ and $u_{\max }=10 K_{u}$. 

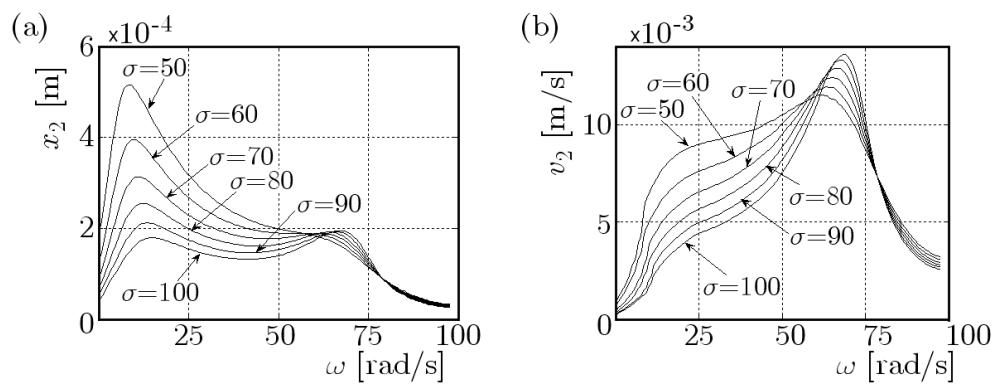

Fig. 10. SMC regulation - effect of parameter $\sigma\left(\beta_{x}=\beta_{v}=1, \gamma=0.1\right)$ : (a) velocity $v_{2}$, (b) momentary power
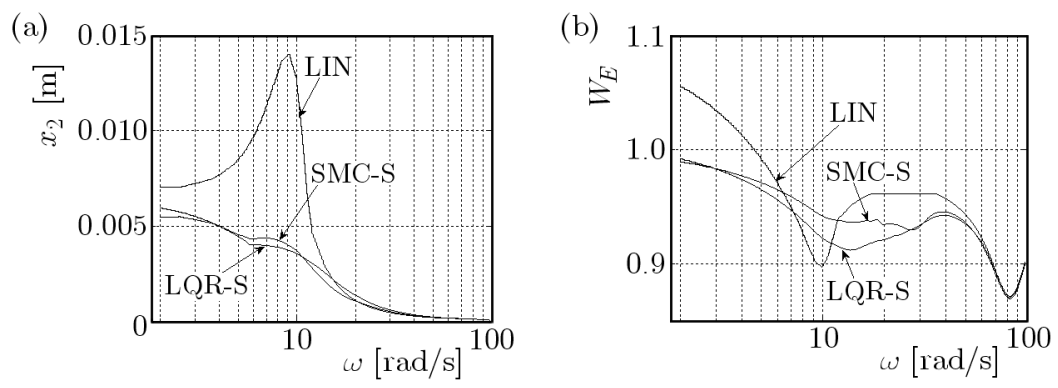

Fig. 11. Comparison of semi-active LQR-S, SMC-S and passive LIN system $\left(\sigma=100 \mathrm{~s}^{-1}\right)$ :

(a) displacement $x_{2}$, (b) Eusam index $W_{E}$

It needs to be pointed out that the value of the parameter $\alpha_{x}$ for LQR-S is different from the one used for LQR. For the previously used parameters $\alpha_{x}, \alpha_{v}$ and $\rho$, the system with LQR-S controller does not behave well in the low-frequency regime (below to $60 \mathrm{rad} / \mathrm{s}$ ). The SMC-S regulator works well when the parameters $\beta_{x}$ and $\gamma$ are low, but the effect of the parameter $\beta_{x}$ is less significant than in the case of active control - that is why the same value as for the SMC is used in the calculations.

Both semi-active systems reduce the amplitude of displacement to the same degree. Although both areless effective in the minimization of vibration when compared to the active systems, but when compared in terms of driving safety both are better due to much higher minimum values of the Eusam index.
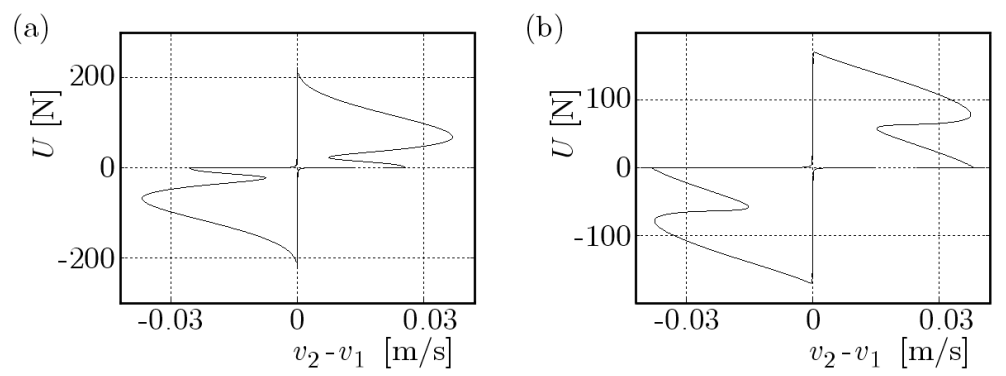

Fig. 12. Control force characteristic $\left(\omega=8.396 \mathrm{rad} / \mathrm{s}\right.$ and $\left.a=5 \mathrm{~mm}, \sigma=100 \mathrm{~s}^{-1}\right)$ : (a) LQR-S, (b) SMC-S

The similar operation of LQR-S and SMC-S, is most probably the effect of similar force characteristics generated by both regulators. Figures 12 and 13 show the relation between the control force and the relative velocity for LQR-S $\left(\alpha_{x}=\alpha_{v}=1, \rho=0.1\right)$ and SMC-S $\left(\beta_{x}=\beta_{v}=1, \gamma=0.1\right)$ systems.

It is assumed that the system is exposed to harmonic excitation defined by the parameters: $\omega=8.396 \mathrm{rad} / \mathrm{s}$ and $a=0.005 \mathrm{~m}$ (first resonance) and $\omega=84.39 \mathrm{rad} / \mathrm{s}$ and $a=0.0004 \mathrm{~m}$ 
(a)

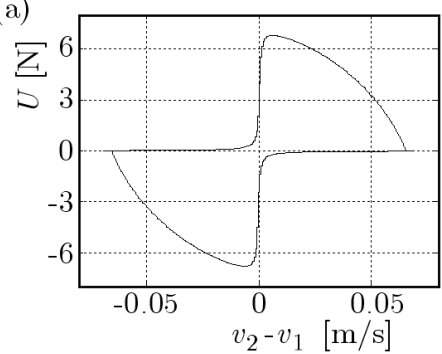

(b)

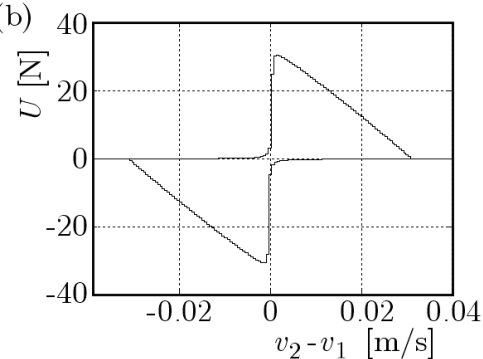

Fig. 13. Control force characteristic $\left(\omega=84.39 \mathrm{rad} / \mathrm{s}, a=0.4 \mathrm{~mm}, \sigma=100 \mathrm{~s}^{-1}\right)$ : (a) LQR-S, (b) SMC-S

(second resonance). The plot of the characteristics in the first and third quadrant of the system is due to the condition imposed on the momentary power. A less-than-ideal plot of the curve in those quadrants is the result of replacing the discontinuous function (5.2) by a continuous one. For the angular frequency $\omega=8.396 \mathrm{rad} / \mathrm{s}$, both characteristics are similar qualitatively and quantitaviley. For the second resonance, the differences are more significant with the visibly higher control force values of SMC-S.

\section{Conclusions}

From the analysis of the results and observations obtained from numerical simulations performed under different conditions, several conclusions can be drawn:

- The application of both active systems, especially the SMC algorithm, greatly reduces the amplitude, velocity and acceleration within the frequency range close to the first resonance, which in turn slightly increases the comfort of passengers during driving.

- Active systems are however unsatisfactory, within the range of the second resonance frequency, as they decrease the Eusam index describing how well the wheel-road adhesion is, and also increase the momentary power, and so the energy that needs to be introduced to the system.

- The analyzed systems, especially the active controllers, are sensible to time delay between the regulator and the actuator. This fact should be taken into consideration when choosing the parameters of the regulator.

- The semi-active systems are visibly less effective within the region of the first resonance frequency, however due to their lower cost and higher resistance to control signal time delays, they can become an alternative to the active systems.

\section{References}

1. Ahmadian M., Vahdati N., 2006, Transient dynamics of semiactive suspensions with hybrid control, Journal of Intelligent Material Systems and Structures, 17, 2, 145-153

2. Chen P.-C., Huang A.-C., 2005, Adaptive sliding control of non-autonomous active suspension systems with time-varying loadings, Journal of Sound and Vibration, 282, 1119-1135

3. Fischer D., Isermann R., 2004, Mechatronic semi-active and active vehicle suspensions, Control Engineering Practice, 12, 1353-1367

4. Huang S.-J., Chen H.-Y., 2006, Adaptive sliding controller with self-tuning fuzzy compensation for vehicle suspension control, Mechatronics, 16, 607-622

5. Lin J., Lian R.-J., Huang C.-N., Sie W.-T., 2009, Enhanced fuzzy sliding mode controller for active suspension systems, Mechatronics, 19, 1178-1190 
6. Liu Y., Waters T.P., Brennan M.J., 2005, A comparison of semi-active damping control strategies for vibration isolation of harmonic disturbances, Journal of Sound and Vibration, 280, 21-39

7. Łuczko J., 2011, Comparison of dynamical responses of semi-active dampers described by the Bouc-Wen and Spencer models (in Polish), Czasopismo Techniczne, 2, 1-M 127-136

8. Łuczko J., Ferdek U., 2012, Comparison of different control strategy in semi-active vehicle suspension system (in Polish), Czasopismo Techniczne, 11, 6-M, 81-92

9. Maciejewski I., 2012, Control system design of active seat suspensions, Journal of Sound and Vibration, 331, 1291-1309

10. Makowski M., Knap L., Grzesikiewicz W., 2011, Modelowanie i identyfikacja parametrów sterowanych tłumików magnetoreologicznych, Modelowanie Inżynierskie, 41, 261-269

11. Orman M., Snamina J., 2009, Comparison of effectiveness of semi-active and active cable vibration dampers (in Polish), Czasopismo Techniczne, 12, 3-M, 71-83

12. Rajeswari K., Lakshmi P., 2008, GA tuned distance based fuzzy sliding mode controller for vehicle suspension systems, International Journal of Engineering and Technology, 5, 1, 36-47

13. Rao LVV Gopala, NARAyAnAn S., 2009, Sky-hook control of nonlinear quarter car model traversing rough road matching performance of LQR control, Journal of Sound and Vibration, 323, 515-529

14. Sam Y.M., Osman J.H.S., 2005, Modeling and control of the active suspension system using proportional integral sliding mode approach, Asian Journal of Control, 7, 2, 91-98

15. Sam Y.M., Suaib N.M., Osman J.H.S., 2008, Hydraulically Actuated Active Suspension System with Proportional Integral Sliding Mode Control, WSEAS Transactions on Systems and Control, $\mathbf{9}, 3,859-868$

16. Sapiński B., RosóŁ M., 2008, Autonomous control system for a 3 DOF pitch-plane suspension model with MR shock absorbers, Computers and Structures, 86, 379-385

17. Snamina J., Kowal J., Wzorek T., 2011, Analysis of the energy dissipation in vehicle suspensions for selected control algorithm (in Polish), Czasopismo Techniczne, 2, 1-M, 233-240

18. Tomera M., 2010, Nonlinear controller design of a ship autopilot, International Journal of Applied Mathematics and Computer Science, 20, 2, 271-280

19. Wu X., GRIffin M.J., 1997, A semi-active control policy to reduce the occurrence and severity of end-stop impacts in a suspension seat with an electrorheological fluid damper, Journal of Sound and Vibration, 203, 5, 781-793

20. YILDIRIM S., 2004, Vibration control of suspension systems using a proposed neural network, Journal of Sound and Vibration, 277, 1059-1069

21. Yoshimura T., Kume A., Kurimoto M., Hino J., 2001, Construction of an active suspension system of a quarter car model using the concept of sliding mode control, Journal of Sound and Vibration, 239, 2, 187-199 